International Archives of the Photogrammetry, Remote Sensing and Spatial Information Sciences, Volume XL-7/W1, 3rd ISPRS IWIDF 2013, 20 - 22 August 2013, Antu, Jilin Province, PR China

\title{
RESEARCH OF MULTI-BASELINE INSAR ELEVATION INVERSION BASED ON MLHE METHOD
}

\author{
Hua Fenfen ${ }^{\mathrm{a}, \mathrm{b}}$, Zhang Jixian ${ }^{\mathrm{b}}$, Huang Guoman ${ }^{\mathrm{b}}$ \\ ${ }^{a}$ School of Environmental Science and Spatial Informatics, China University of Mining and Technology Xuzhou, \\ China, bthree@tom.com \\ ${ }^{\mathrm{b}}$ Institute of Photogrammetry and Remote Sensing Chinese Academy of Surveying and Mapping, Beijing, China
}

KEY WORDS: Multi-Baseline InSAR; Maximum Likelihood Height Estimate; Probability Density Function; Phase error

\begin{abstract}
:
In this paper, we studied on Maximum Likelihood Height Estimate (MLHE) method to invert elevation using Multi-Baseline InSAR (MB-InSAR) Data. Then a set of simulated data were adopted to do the experiment to illustrate its availability. Meanwhile, this paper also presents the advantages and disadvantages of this approach with analysis of the influence of phase, baseline and range error on elevation inversion, as well as the following attention.
\end{abstract}

\section{INTRODUCTION}

InSAR technology makes it possible for topographic mapping by using phase information recorded by SAR data to obtain terrain 3D information. Based on this, Multi-Baseline InSAR (MB-InSAR) introduces coherent coefficient as the interferometric phase index and uses multiple interferograms to invert elevation, which not only avoids the task of phase unwrapping, but considerably reduces the influence of noise. Therefore, this paper studied on Maximum Likelihood Height Estimate (MLHE) method to invert elevation using MB-InSAR Data and presents some important conclusions.

\section{MLHE METHOD}

Elevation inversion based on MLHE method makes the best of the phase and amplitude information in SAR images without the assumption that the phase difference between adjacent pixels must be less than $\pi$. So, this method has better adaptability and high precision.

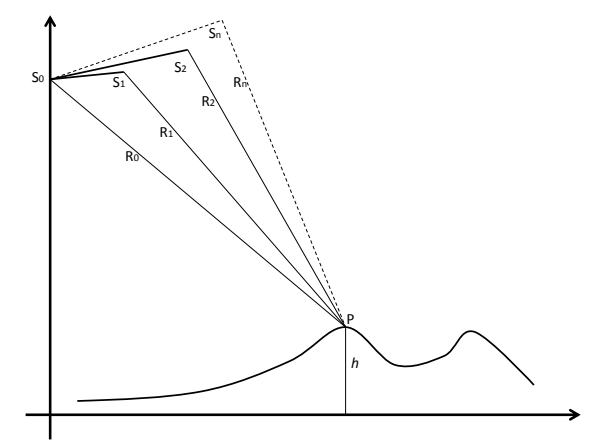

Fig 1. MB-InSAR geometry model

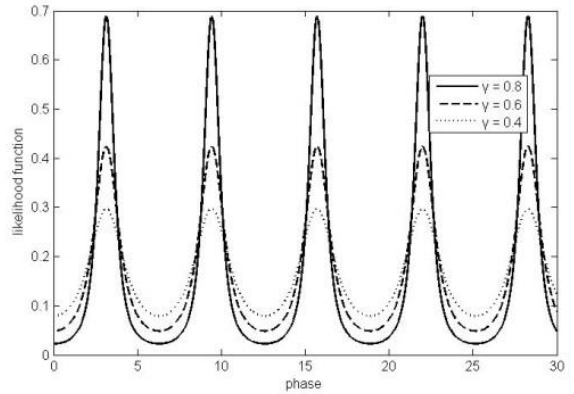

Fig 2. PDF of different coherence coefficients 0.8, 0.6, 0.4

As shown in Fig $1, \mathrm{~S}_{0}, \mathrm{~S}_{1}, \mathrm{~S}_{2}, \cdots \cdots, \mathrm{S}_{\mathrm{n}}$ are $\mathrm{SAR}$ platforms position, and $\mathrm{R}_{0}, \mathrm{R}_{1}, \mathrm{R}_{2}, \cdots \cdots, \mathrm{R}_{\mathrm{n}}$ represents the distance between SAR sensors and target P. Any two of $\mathrm{n}+1$ SAR images can form an interferogram. If we assume $S_{0}$ as master image, the theoretical interferometric phase in the interferogram can be expressed by (1).

$$
\phi_{h}(i)=\left\langle\frac{4 \pi}{\lambda}\left(R_{0}-R_{i}\right)\right\rangle_{2 \pi}
$$

Where $\lambda$ is radar wavelength, $\langle\cdot\rangle_{2 \pi}$ is the remainder after dividing $2 \pi$. In actual operation, (1) can be written as follows.

$$
\phi_{h}(i)=\left\langle\mathrm{g}\left(R_{0}, \overrightarrow{B_{l}}, h\right)\right\rangle_{2 \pi}
$$

The probability density function describes the relation of measured phase and theorical phase. 


$$
\begin{gathered}
f\left(\phi(i) \mid \phi_{h}(i)\right)=\frac{1}{2 \pi} \frac{1-|\gamma|^{2}}{1-|\gamma|^{2} \cos ^{2}\left(\phi(i)-\phi_{h}(i)\right)} \\
\times\left\{1+\frac{|\gamma| \cos \left(\phi(i)-\phi_{h}(i)\right) \cos ^{-1}\left[-|\gamma| \cos \left(\phi(i)-\phi_{h}(i)\right)\right]}{\sqrt{1-|\gamma|^{2} \cos ^{2}\left(\phi(i)-\phi_{h}(i)\right)}}\right\} \\
\phi \in(-\pi, \pi]
\end{gathered}
$$

Where $\gamma$ is coherence coefficient. Considering the relationship between interferometric phase and elevation, we can obtain the probability density function of elevation shown in Fig 2 from (2) and (3).

$$
\begin{aligned}
& f(\phi(i) \mid h)=\frac{1}{2 \pi} \frac{1-|\gamma|^{2}}{1-|\gamma|^{2} \cos ^{2}\left(\phi(i)-g\left(R_{0}, \overrightarrow{B_{l}}, h\right)\right)} \\
& \times\left\{1+\frac{|\gamma| \cos \left(\phi(i)-g\left(R_{0}, \overrightarrow{B_{l}}, h\right)\right) \cos ^{-1}\left[-|\gamma| \cos \left(\phi(i)-g\left(R_{0}, \overrightarrow{B_{l}}, h\right)\right)\right]}{\sqrt{1-|\gamma|^{2} \cos ^{2}\left(\phi(i)-g\left(R_{0}, \overrightarrow{B_{l}}, h\right)\right)}}\right\}
\end{aligned}
$$

Elevation inverted by MLHE method corresponds to elevation obtained when (4) is in maximum value, that is the maximum likelihood solution of target P's elevation.

$$
\hat{h}_{M L}=\arg \max _{h} f(\phi(i) \mid h)
$$

Due to the interferometric phase is a remainder with integer ambiguities, the likelihood function has multiple peak positon as shown in Fig 2 corresponding to infinite $\hat{h}_{M L}$.

$$
\hat{h}_{M L}(i, j)=\frac{\lambda \phi}{4 \pi d} \pm k \frac{\lambda}{2 d}, \quad k=0,1,2, \cdots \cdots
$$

And every interferogram has a likelihood function, then (4) and (5) can be written as

$$
\begin{aligned}
& F_{m f}(\Phi \mid h)=\prod_{i=1}^{n} f(\phi(i) \mid h) \\
& \hat{h}_{M L}=\arg \max _{h} F_{m f}(\Phi \mid h)
\end{aligned}
$$

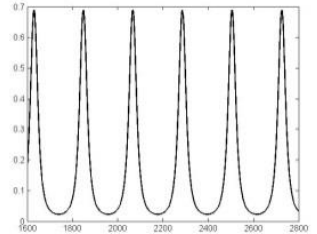

(a)

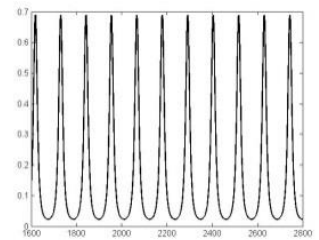

(c)

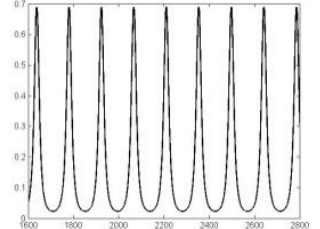

(b)

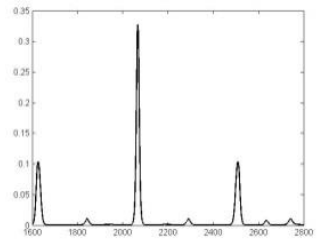

(d)
Fig 3. The likelihood functions in different baseline cases and MB-InSAR PDF (a) short baseline (b) general baseline (c)long baseline (d) multiplied result by PDFs

As can be seen from Fig 3, different baseline length corresponds to different likelihood function. If we multiply PDFs, we can acquire the very peak position in a rational elevation interval calculated by external DEM or other ways.

\section{RESULTS WITH MB-INSAR DATA}

This paper performs the experiment with the simulation data and gets some significant conclusions. Simulation data consists of TerraSAR-X system parameter and slant range elevation image generated by SRTM. Based on the same antenna and baseline position, we can separately get the simulation interferograms and elevation images without no noises and errors in different baselines (19m, 29m and 37m) and by MLHE method.

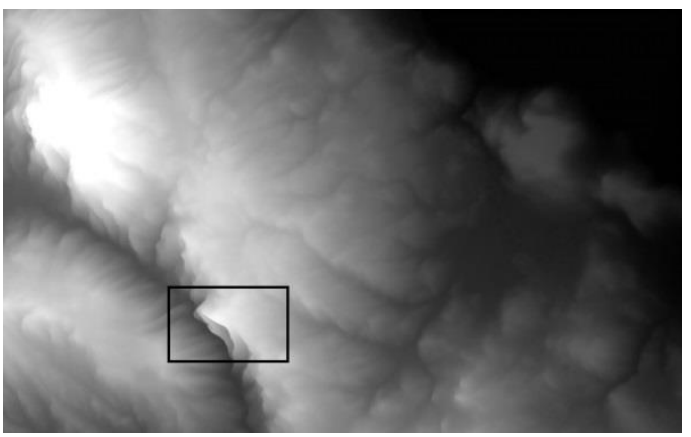

(a)

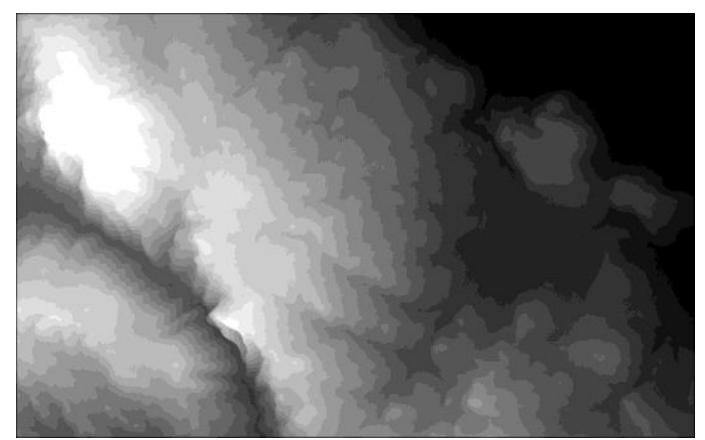

(b)
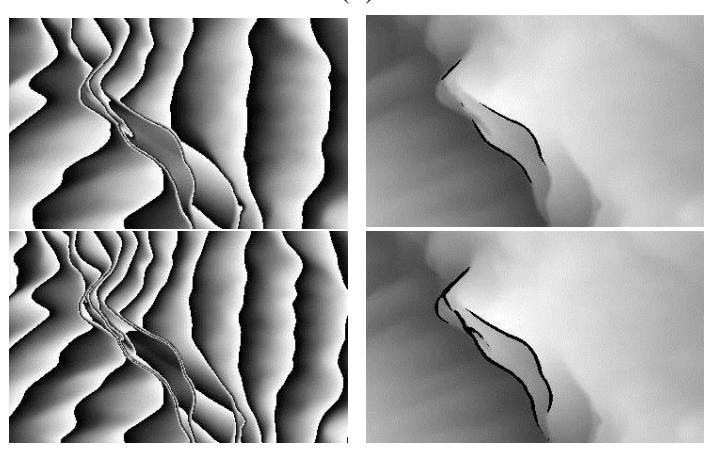


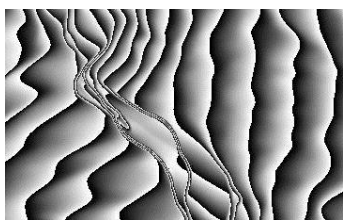

(c)

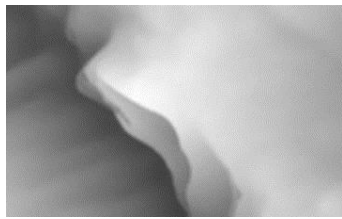

(e)

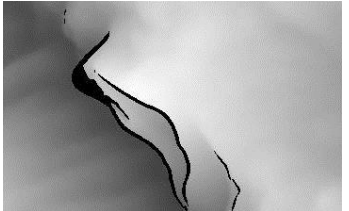

(d)

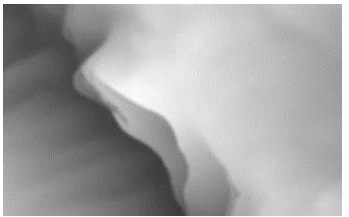

(f)
Fig 4. Simulation Results (a) Reference slant-range elevation image (b) Inversion elevation image by MLHE method (c) Simulation interferograms in different baselines (d) Invesion elevation images in different baselines (e) Inversion elevation image by MLHE (f) Reference slant-range elevation image

We focus on part of experimental area, the rectangular region shown in Fig 4(a) for convenience's sake. Fig 4(c) and Fig 4(d) shows the interferograms and inversion elevation images of interested region in different baselines, and we can see that with the baseline growth, the interference fringe becomes more and more intensive on the hillside facing the radar sensor, so more unwrapping area appears. However, Fig 4(e) displays perfect inversion elevation without blank by MLHE almost the same with reference slant-range elevation image in Fig 4(f).

The following process should calculate statistical error. Where M,N separately represents number of rows and columns, and we use $h(m, n)$ and $\operatorname{dem}(m, n)$ in place of inversion and true elevation value, that are known from elevation images.

$h_{e r r}=\sqrt{\frac{1}{M \times N} \sum_{n=1}^{N} \sum_{m=1}^{M}(\operatorname{dem}(m, n)-h(m, n))^{2}}$

\begin{tabular}{|l|c|l|c|}
\hline INSTRUCTION & ERROR & INSTRUCTION & ERROR \\
\hline Reference & $0.00 \mathrm{~m}$ & Baseline error & $4.04 \mathrm{~m}$ \\
elevation image & & $0.001 \mathrm{~m}$ & \\
Single baseline & $3.83 \mathrm{~m}$ & Baseline error & 156.95 \\
with noises & & $0.01 \mathrm{~m}$ & $\mathrm{~m}$ \\
With no noise & $1.1 \mathrm{e}-6$ & Slant range error & $0.90 \mathrm{~m}$ \\
& $\mathrm{~m}$ & $1 \mathrm{~m}$ & \\
With noises & $17.61 \mathrm{~m}$ & Slant range error & $9.00 \mathrm{~m}$ \\
& & $10 \mathrm{~m}$ & \\
With noises and & $12.77 \mathrm{~m}$ & Slant range error & $90.04 \mathrm{~m}$ \\
filtered & & $100 \mathrm{~m}$ & \\
\hline
\end{tabular}

Table 1. Statistical errors of simulation data
Single baseline condition is the baseline 3 cases, $37 \mathrm{~m}$. Random noise is the mean of Orad, the standard deviation of $0.1 \mathrm{rad}$, distributed in the range $[-\pi, \pi]$.

As can be seen from the table, in the case of no the system parameter error, no phase noise, the algorithm can obtain very high accuracy values of elevation without ground control points. The noise had a greater influence on elevation accuracy, than the single baseline condition. Effect of phase error of the single baseline case is approximately linear, multi-baseline case more complex. In the simulation above, we assume that the baseline 1 and baseline 2 with no phase error, phase error effect on baseline 3 distributed in $[-\pi, \pi]$. the results are as follows:

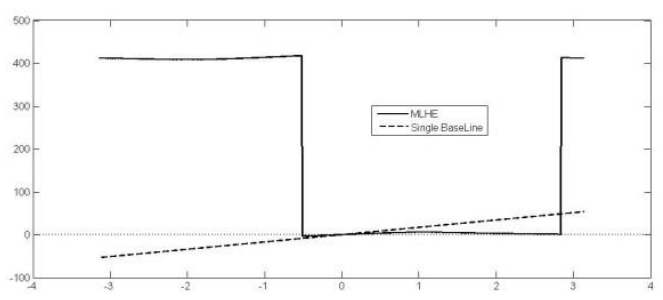

(a)

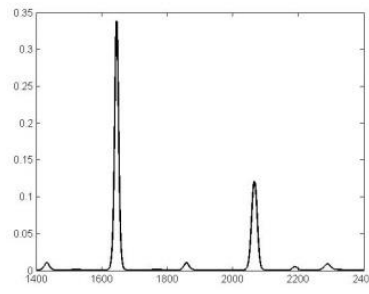

(b)

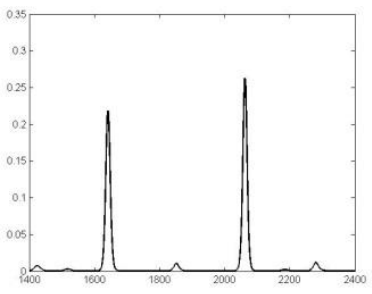

(c)
Fig 5. Effect of phase error of elevation in the case of single baseline and multi-baseline

In Fig 5(a), when the phase error is within a certain range, impact on multi-baseline is much lower than single baseline, but beyond a certain range, impact on multi-baseline will be mutated to tens even hundreds of times. This is because when the phase error being changed, the PDF shifted and the maximum of the product of multiple PDFs will transfer from one extreme to another extreme, Fig 5 (b)(c).

Influence of baseline error of elevation inversion is very serious. Slant range error of elevation inversion effect of approximate constant.

\section{CONCLUSIONS}

Multi-baseline interferometric SAR has many advantages than single baseline: 1) have a certain ability to resist noise, when the noise is small; 2) can estimate altitude value from interferometric phase directly, without phase unwrapping and ground control point; 3) can handle surface discontinuities.

The experiment found that small baseline error will cause the elevation error, or even lead to vertical wavy distribution. Therefore system parameters need to be calibrated. 
International Archives of the Photogrammetry, Remote Sensing and Spatial Information Sciences, Volume XL-7/W1, 3rd ISPRS IWIDF 2013, 20 - 22 August 2013, Antu, Jilin Province, PR China

\section{References:}

Ferraiuolo, G., Pascazio, V. 2002. A Bayesian Approach Based on Modified Markov Random Fields for Microwave Tomography. IGARSS'02, pp. 3393 3395.

Ferraiuolo, G., Pascazio, V., Schirinzi, G. 2004a. Maximum a Posteriori Estimation of Height Profiles in InSAR Imaging. IEEE Trans. on GRS, 1(2), pp. 66-70.

Ferraiuolo, G., Leva, D., Nico, G., et al. 2004b. A Bayesian Technique for Terrain Mapping Using Multi-frequency Ground Based Interferometric SAR Systems. IGARSS'04, pp. 469-472.

Lee, J. S., Hoppel, K. W., Mango, S. A., et al. 1994. Intensity and Phase Statistics of MultilookPolarimetric and Interferometric SAR Imagery. IEEE Transactions on Geoscience and Remote Sensing, 32(5), pp. 1017-1028

Li, F., Bao, Z., Li, H., et al. 2006. Image Autocoregistration and InSAR Interferogram Estimation Using Joint Subspace Projection. IEEE Transactions on Geoscience and Remote Sensing, 44(2), pp. 288-297

Lombardini, F., 1996. Absolute Phase Retrieval in a Three-element Synthetic Aperture Radar Interferometer. CIE International Conference of Radar, pp. 309-312.
Pascazio, V., Schirinzi, G. 2001. Estimation of Terrain Elevation by MultifrequencyInterferometric Wide Band SAR Data. IEEE Signal Processing Lett, 8 pp. 7-9.

Pascazio, V., Schirinzi G. 2002. Multifrequency InSAR Height Reconstruction through Maximum Likelihood Estimation of Local Planes Parameters. IEEE Trans. on Image Processing, 11(12), pp. 1478-1489.

Schmitt, M., Schmitt, U. 2013. Compressive Sensing Based Layover Separation in Airborne Single-Pass Multi-Baseline InSAR Data. IEEE Transactions on Geoscience and Remote Sensing, 51(3), pp. 313-317.

Schmitt, M., Schmitt, U. 2012. Layover Separation in Airborne Single Pass Multi-baseline InSAR Data Based on Compressive Sensing. EUSAR 2012, pp. 30-33.

Xu, W., Chang, C., Kwoh, L. K., et al. 1994. Phase Unwrapping of SAR Interferogram with Multi-Frequency or Multi-Baseline. IGARSS'94, pp. 730-732.

Zhang, L., Wang, F. 2006. Improving the Interferometric Phase Accuracy of Distributed Satellites InSAR System with Multibaseline Data Fusion. Journal of Electronics \& Information Technology, 28(11), pp. 2011-2014. 